\title{
Frailty and healthcare utilisation across care settings among community-dwelling older adults in Singapore
}

\author{
Lixia Ge ${ }^{1 *}$, Chun Wei Yap', Bee Hoon Heng ${ }^{1}$ and Woan Shin Tan ${ }^{1,2}$
}

\begin{abstract}
Background: Frailty is frequently found to be associated with increased healthcare utilisation in western countries, but little is known in Asian population. This study was conducted to investigate the association between frailty and healthcare utilisation in different care settings among community-dwelling older adults in Singapore.

Methods: Data from a population health survey among community-dwelling adults were linked with an administrative database to retrieve data of healthcare utilisation (including government primary care clinic visits, specialised outpatient clinic visits, emergency department visits, day surgery and hospitalisations) occurred during a six-month look-back period and sixmonth post-baseline respectively. Baseline frailty status was measured using the five-item FRAll scale, which was categorised into three groups: robust (0), pre-frail (1-2), and frail (3-5). Negative binomial regression was applied to examine the association between frailty with respective healthcare utilisation (dependent variables), controlling for other confounding variables.
\end{abstract}

Results: In our sample of 701 older adults, $64.8 \%$ were of robust health, $27.7 \%$ were pre-frail, and $7.6 \%$ were frail. Compared to the robust group, frail individuals had a higher rate of specialised outpatient clinic visits (incidence rate ratio (IRR): 2.8, 95\% confidence interval (Cl): 1.2-6.5), emergency department visits (IRR: 3.1, 95\%Cl: 1.1-8.1), day surgery attendances (IRR: 6.4, 95\%Cl: 1.3-30.9), and hospitalisations (IRR: 6.7, 95\%Cl: 2.1-21.1) in the six-month period prior to the baseline and in subsequent 6 months (IRR: 3.3, 95\%Cl: 1.6-7.1; 6.4, 2.4-17.2; 5.8, 1.3-25.8; 13.1, 4.9-35.0; respectively), controlling for covariates.

Conclusions: Frailty was positively associated with the number of specialised outpatient clinic visits, emergency department visits, day surgeries and hospitalisations occurred during 6 months prior to and after the baseline. As frailty is a potentially reversible health state with early screening and intervention, providing preventive activities that delay the onset or progression of frailty should have potential effect on delaying secondary and tertiary care utilisation.

Keywords: Frailty, Healthcare utilisation, Community-dwelling older adults

\footnotetext{
*Correspondence: lixia_ge@nhg.com.sg

'Health Services \& Outcomes Research, National Healthcare Group Pte Ltd, 3

Fusionopolis Link, \#03-08 Nexus@one-north (South Lobby), Singapore 13854, Singapore

Full list of author information is available at the end of the article
}

(c) The Author(s). 2020 Open Access This article is licensed under a Creative Commons Attribution 4.0 International License, which permits use, sharing, adaptation, distribution and reproduction in any medium or format, as long as you give appropriate credit to the original author(s) and the source, provide a link to the Creative Commons licence, and indicate if changes were made. The images or other third party material in this article are included in the article's Creative Commons licence, unless indicated otherwise in a credit line to the material. If material is not included in the article's Creative Commons licence and your intended use is not permitted by statutory regulation or exceeds the permitted use, you will need to obtain permission directly from the copyright holder. To view a copy of this licence, visit http://creativecommons.org/licenses/by/4.0/. The Creative Commons Public Domain Dedication waiver (http://creativecommons.org/publicdomain/zero/1.0/) applies to the data made available in this article, unless otherwise stated in a credit line to the data. 


\section{Background}

Frailty can be defined as 'a state of vulnerability to adverse outcomes resulting from the accumulation of deficits associated with clinical effects' [1], and has been shown to be a common phenomenon among older adults [2]. A recent systematic review that gathered data from 21 studies and over 61,500 community-dwelling older adults found that the overall weighted average prevalence of frailty was $10.7 \%$ (range: $4.0-59.1 \%$ ) [2]. With the absolute number of people aged 60 years and over expected to reach 2 billion in 2050 [3], the burden of frailty will increase [4].

In Singapore, $21.4 \%$ of the total population consisted of individuals aged 60 years and above in 2019 [5], which is projected to reach $40 \%$ by year 2050 [6]. Such drastic rise in both the number and proportion of older population inevitably translate into a surge in the number and proportion of frail individuals [7], which brings various challenges to health care and healthcare delivery as they are recognised as intensive users of health care services [8]. To forge a frailty-ready healthcare system, Singapore has re-organised its public healthcare system from six regional healthcare systems (RHS) into three integrated clusters to allow each cluster to have a fuller range of assets, capabilities, services and networks across different care settings to meet the challenges of population ageing and further care needs [9]. Each RHS is responsible for care integration and providing care to the population in a specific geographical region. Innovative projects have been implemented in each RHS to address the needs of the frail elderly in Singapore [10] but a deeper understanding of the frail older population and their patterns of healthcare utilisation is necessary for better resource planning and intervention prioritisation in public healthcare.

Based on self-reported incidence of healthcare resource utilisation, cross-sectional studies in international literature have found frailty to be associated with an increased likelihood of general practice (adjusted odds ratio (OR): 2.1-4.4), specialist (OR: 1.3-1.8), emergency department (OR: 2.5-6.2) and inpatient (OR: 2.1-3.3) service utilisation $[8,11-13]$. These findings were also supported by the results from prospective cohort studies [14-16] and panel studies [17]. It is recogonised that most studies investigating the associations between frailty and healthcare utilisation were conducted in North American and European countries. As healthcare systems and access to care varies across countries, examining the association between frailty and healthcare utilisation using Singapore data will provide insights in an Asian setting where health seeking behaviours and utilisation patterns could differ.

As the assessment of frailty are typically determined based on the actual or estimated status of the person at the point of assessment, it usually does not account for the presence of acute conditions which might sway the determination of frailty status [18]. This will contribute to the variation in the magnitude of association between frailty and healthcare utilisation in different settings. While individuals who have been frail over a period may have persistently higher healthcare utilisation during retrospective and prospective observation periods, those with frailty caused by transient conditions might only have temporarily higher healthcare utilisation for a short period of time. In prior studies, the association between frailty and healthcare utilisation was explored using either retrospective or prospective data in different population. There is a scarcity of research that examined their associations using both retrospective and prospective utilisation data. As such, little is known about how the magnitude of association differs in different periods of time. This study aims to investigate the association of frailty and healthcare utilisation in community-dwelling older adults aged 60 years and above, with utilisation data collected in two different periods of time: 1) 6 months prior to the baseline frailty assessment, and 2) 6 months after the baseline assessment.

\section{Methods \\ Study participants}

Older adults aged 60 years and over $(n=701)$ who agreed to use their National Registration Identity Card (NRIC) number to link with administrative database to retrieve healthcare utilisation data were sampled from the Population Health Index (PHI) study, a population-based health survey conducted in the Central Region of Singapore. The baseline data of PHI which were collected during November 2015 to November 2016 were used for this study. The sampling procedure and survey methodology of the baseline PHI study has been described elsewhere [19-21]. In brief, eligible participants (Singapore citizens or permanent residents, aged 21 years and above and lived in the selected housing unit for the past 6 months) staying in randomly selected household units in the Central Region of Singapore were identified via doorto-door visits by trained surveyors and one eligible household member was randomly selected using Kish grid [22]. There were 1942 eligible communitydwelling adults recruited and underwent detailed structured interviews for the baseline PHI survey.

The PHI study was approved by the ethics review committee of the National Healthcare Group (NHG) Domain Specific Review Board (Reference Number: 2015/00269). Written informed consent was obtained from all individual participants after they were fully informed of the study objectives and procedures. 


\section{Frailty assessment}

Frailty was determined using the revised five-item FRAI L scale (Fatigue, Resistance, Ambulation, Illnesses, \& Malnutrition) with the "Malnutrition" replacing the "Loss of weight" in the original FRAIL scale (Fatigue, Resistance, Ambulation, Illnesses, \& Loss of weight) [23, 24]. Each item is scored either 0 or 1 . The revised FRAI $\mathrm{L}$ scale is scored from 0 (best) to 5 (worst) and is translated into three categories: robust (0), pre-frail (1-2), and frail (3-5). Similar to other studies [23, 24], we operationalised the FRAIL scale based on information obtained from specific questions included in the PHI survey questionnaire. "Fatigue" was measured by asking how often they felt tired with responses of "more than half the days" or "nearly every day" scored 1. "Resistance" was assessed by asking their difficulty in walking up and down one flight of stairs without using handrail, and "Ambulation" was measured by asking their difficulty in walking around one floor of home or several blocks without aids; "quite a lot" or "cannot do" responses were each scored as 1. "Illness" was scored 1 for those who reported 5 or more illnesses out of 14 illnesses. "Malnutrition" was scored 1 if Body Mass Index $(\mathrm{BMI})<18.5$ or MNA screening score $<8$ or Mini Nutritional Assessment (MNA) total score $<17$. A complete description of the revised FRAIL scale items' scoring criteria is provided in Table 1.

\section{Healthcare utilisation}

The healthcare utilisation data during the retrospective 6-month and prospective 6-month periods were obtained from RHS database [25]. The RHS database contains linked NHG polyclinic visit records, specialist outpatient clinic (SOC) visit records, emergency department (ED) attendance records, day surgery (DS) attendance records and hospital discharge records from three government hospitals - Tan Tock Seng Hospital, Khoo Teck Puat Hospital and Institute of Mental Health,

Table 1 FRAIL scale items

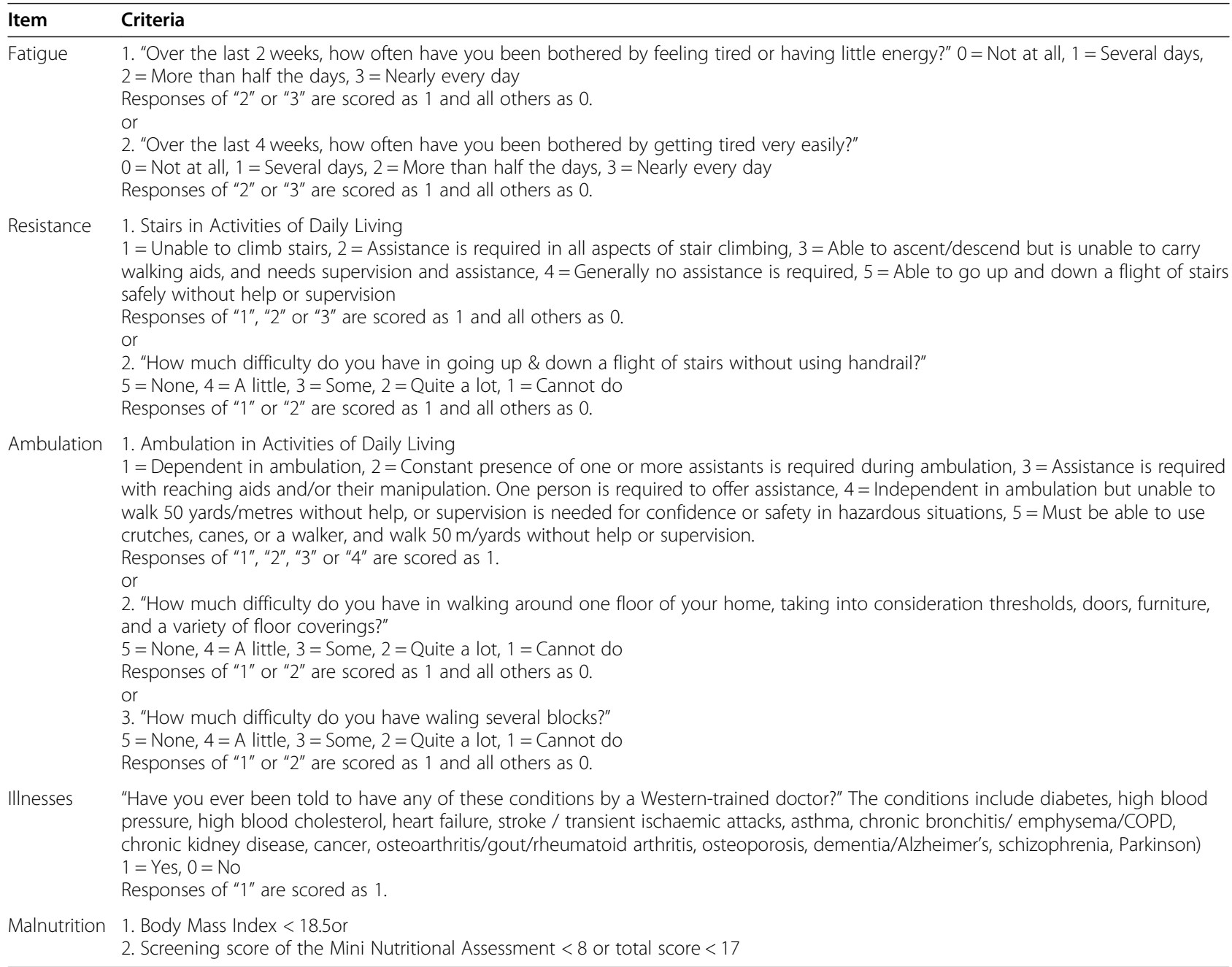


chronic disease management system records and mortality records from local registries. The healthcare utilisation data were categorised according to the main healthcare settings into polyclinic visits, SOC visits, ED visits, DS attendances and hospitalisations. Polyclinic visits refer to doctor consultation and technical visits made by the individual to any of the nine linked NHG polyclinics. SOC visits and ED visits refer to visits to the specialists in outpatient clinics and the emergency rooms located within three government hospitals, respectively. Similarly, DS attendances refer to surgical procedures performed in day surgery rooms where patients were discharged on the same day without admitting to inpatient wards; and hospitalisations refer to inpatient episodes with at least one overnight stay at these hospitals. The survey data and healthcare utilisation data were linked using NRIC numbers which were removed thereafter for data analysis.

\section{Other variables}

We controlled for the confounding effects of covariates to examine the independent effect of frailty on the rates of healthcare utilisation in different care settings. These covariates included demographic factors (age, gender (male / female), Chinese (yes / no), marital status (single / married / widowed or divorced), living arrangement (alone / with others)) $[8,26]$ and smoking status (non-smoker / past smoker / current smoker) [17]. Highest education level (no formal education / primary / secondary or above) and selfperceived money sufficiency for basic living needs (sufficient / insufficient) were also included as control variables as they are enabling factors which influence individuals' health seeking behaviours and healthcare utilisation $[8,27,28]$.

Multimorbidity and disability, which are related to but also distinct from frailty $[29,30]$, were commonly adjusted in studies examining the association between frailty and healthcare utilisation $[8,14]$. We controlled for multimorbidity as a dichotomous variable (yes / no) which was defined as the presence of two or more of the following 17 chronic conditions: dyslipidemia, high blood pressure, diabetes, chronic kidney disease (CKD), heart attack / ischemic heart disease, heart failure, stroke / transient ischemic attack, asthma, chronic bronchitis / emphysema / chronic obstructive pulmonary disease (COPD), cancer, osteoarthritis / gout / rheumatoid arthritis, osteoporosis, depression, anxiety disorder, schizophrenia, dementia / Alzheimer's, and Parkinson's disease [20] (The prevalence of individual chronic diseases among the study participants is presented in the Supplementary Table S1). Disability, which was determined based on whether assistance was required in any of the ten activities of daily living (ADLs) (yes / no) measured using the Modified Barthel Index [31], was also controlled in the models.

\section{Statistical analysis}

Characteristics of the study population were described using mean and standard deviation (SD) for continuous variables, and frequency and percentages for categorical variables. Mean and SD were used to describe healthcare utilisation in every frailty group. To examine the differences in characteristics and utilisation across frailty groups, one-way analysis of covariance (ANOVA) tests (normally distributed) or Kruskal-Wallis $\mathrm{H}$ tests (nonnormally distributed) were performed for continuous variables, and chi-squared tests were conducted for categorical variables.

Healthcare utilisation by settings are count variables characterised by a point mass at zero followed by a right-skewed, discrete distribution, and non-negative values [32, 33]. Given the over-dispersion of data (the conditional variance is larger than the conditional mean), a negative binomial distribution was chosen over a Poisson regression [34]. Healthcare utilisation in five settings formed five different dependent variables and were analysed independently; and the three-level frailty category (robust, pre-frail, frail) was the independent variable of interest. We further adjusted for control variables including demographic factors (including age, gender, ethnic group, marital status, and living arrangement), socioeconomic status (highest education level, self-perceived money insufficiency), smoking status, multimorbidity, and disability. The results were presented as incidence-rate ratios (IRRs) and their corresponding 95\% confidence intervals (CIs). All analyses were performed using Stata/SE 16.1. A $p$ value of less than 0.05 was set as the level of significance.

\section{Results}

\section{Characteristics of study population}

Our sample comprised 701 older adults. Their mean age was 70.5 years (SD 8.2). The majority were of Chinese ethnicity (84\%), female (57\%) and were living with others (81\%). The prevalence of multimorbidity and disability (assistance required for any ADLs) among this population was 70 and $15 \%$, respectively. The proportion of pre-frail and frail individuals measured using the revised FRAIL scale was 28 and 7\%, respectively (Table 2).

Comparing the profile across the three frailty groups (Table 2), we showed that frail elderly were significantly older, and had a higher proportion with multimorbidity or required assistance in any ADLs. A significantly lower proportion of frail elderly were single, of Chinese ethnicity; and a higher proportion had no formal education and perceived that they had insufficiency financial means for their daily needs. 
Table 2 Characteristics of participants at baseline by frailty groups, $\mathrm{n}(\%)$

\begin{tabular}{|c|c|c|c|c|c|}
\hline Characteristics & Overall $(N=701)$ & Robust ( $n=454,64.8 \%$ ) & Pre-frail $(n=194,27.7 \%)$ & Frail $(n=53,7.5 \%)$ & $p$-value \\
\hline Age, mean \pm SD & $70.5 \pm 8.2$ & $68.3 \pm 6.6$ & $73.1 \pm 8.6$ & $79.1 \pm 9.8$ & $<0.001$ \\
\hline Female (n, \%) & $397(56.6)$ & $245(54.0)$ & $122(62.9)$ & $30(56.6)$ & 0.111 \\
\hline Chinese & $591(84.3)$ & $398(87.7)$ & $153(78.9)$ & $40(75.5)$ & 0.003 \\
\hline Marital status & & & & & 0.001 \\
\hline Single & $88(12.5)$ & $64(14.1)$ & $21(10.8)$ & $3(5.7)$ & \\
\hline Married & $410(58.5)$ & $279(61.5)$ & $107(55.2)$ & $24(45.3)$ & \\
\hline Divorce/widowed & $203(29.0)$ & $111(24.4)$ & $66(34.0)$ & $26(49.0)$ & \\
\hline Highest education & & & & & $<0.001$ \\
\hline No formal education & $245(35.0)$ & $123(27.1)$ & $92(47.4)$ & $30(56.6)$ & \\
\hline Primary & $124(17.7)$ & $85(18.7)$ & $30(15.5)$ & $9(17.0)$ & \\
\hline Secondary or higher & $332(47.4)$ & $246(54.2)$ & $72(37.1)$ & $14(26.4)$ & \\
\hline Living alone & $131(18.7)$ & $90(19.8)$ & $36(18.6)$ & $5(9.4)$ & 0.185 \\
\hline Self-reported money insufficiency & $113(16.1)$ & $53(11.7)$ & $45(23.2)$ & $15(28.3)$ & $<0.010$ \\
\hline Smoking status & & & & & 0.047 \\
\hline Non-smoker & $527(75.2)$ & $349(76.9)$ & $144(74.2)$ & $34(64.2)$ & \\
\hline Current smoker & $63(9.0)$ & $41(9.0)$ & $19(9.8)$ & $3(5.7)$ & \\
\hline Past smoker & $111(15.8)$ & $64(14.1)$ & $31(16.0)$ & $16(30.2)$ & \\
\hline Multimorbidity & $487(69.5)$ & $276(60.8)$ & $161(83.0)$ & $50(94.3)$ & $<0.001$ \\
\hline Disability & 107 (15.3) & $9(2.0)$ & 58 (29.9) & $40(75.5)$ & $<0.001$ \\
\hline
\end{tabular}

The percentages were reflected as column percentages

\section{Association between frailty and healthcare utilisation Healthcare utilisation in 6-month period prior to the baseline}

Compared to older adults in robust health, significantly higher proportions of SOC and ED visits, DS attendances as well as hospitalisations were observed in those who were pre-frail and frail (Table 3). The mean number of SOC visits, ED visits and hospitalisations also increased corresponding with the increase in frailty levels (all $p<0.001$ ). Pre-frail older adults were the dominant users of the polyclinic services with about $42 \%$ having polyclinic visits.

After adjusting for all covariates including multimorbidity and disability, the negative binomial regression results showed that frailty was statistically associated with the adjusted rates of SOC visits, ED visits, DS attendances and hospitalisations in 6-month period prior to the baseline. Relative to the robust group, individuals who were frail had 2.8 times the rate of SOC visits; had 3.1 times the rate of ED visits; and had a rate 6.4 times and 6.7 times greater for DS attendances and hospitalisations respectively (Table 3). Pre-frail individuals had 1.7 times and 2.1 times the rate of SOC visits and hospitalisations respectively compared to their robust counterpart.

\section{Healthcare utilisation during 6-month period after the baseline}

Similarly, during the 6-month period after the baseline, significantly higher proportion and mean number of
SOC and ED visits, as well as hospitalisations in pre-frail and frail older adults were observed compared to their robust peers (Table 4). After adjusted for all covariates, frail older adults had 3.3 times the rate of SOC visits, 6.4 times the rate of ED visits, and 5.8 times and 13.1 times the rate of DS attendances and hospitalisations respectively compared to their robust counterpart during the 6month period after the baseline. No significant difference in rate was observed for polyclinic visits. Pre-frail individuals had 1.5 times, 2.6 times and 3.8 times higher rate of polyclinic visits, ED visits and hospitalisations respectively compared to their robust counterpart (Table 4).

\section{Discussion}

We examined the associations between frailty and healthcare utilisation in different public healthcare settings among community-dwelling adults aged 60 years and above in Singapore. Although the issue of frailty is predominant among people aged 70 years and above, the public health system in Singapore proactively puts in efforts and resources in identifying the pre-frail individuals and those at risk of frailty, and extends services to people who are aged 60 years and above when designing programmes and interventions. As such, we included participants aged 60 years and above as the study population. The results showed that the association between frailty and healthcare utilisation varied in different 
Table 3 Associations between frailty and healthcare utilisation in different settings during 6-month period prior to the baseline

\begin{tabular}{|c|c|c|c|c|}
\hline Healthcare utilisation by setting & Frailty & Yes, n (\%) & Mean \pm SD & Adjusted IRR ${ }^{\mathrm{C}}(95 \% \mathrm{Cl})$ \\
\hline \multirow[t]{4}{*}{ Polyclinic visits } & Robust $(n=454)$ & $148(32.6)$ & $0.95 \pm 1.96$ & 1.00 \\
\hline & Pre-frail $(n=194)$ & $82(42.3)$ & $1.47 \pm 2.69$ & $1.35(0.96,1.91)$ \\
\hline & Frail $(n=53)$ & $20(37.7)$ & $1.26 \pm 2.03$ & $1.11(0.58,2.10)$ \\
\hline & $p$-value & $0.060^{a}$ & $0.024^{b}$ & \\
\hline \multirow[t]{4}{*}{ Specialist outpatient clinic visits } & Robust $(n=454)$ & $126(27.8)$ & $1.22 \pm 2.93$ & 1.00 \\
\hline & Pre-frail $(n=194)$ & $79(40.7)$ & $2.40 \pm 5.83$ & $1.65(1.04,2.63)$ \\
\hline & Frail $(n=53)$ & $27(50.9)$ & $3.92 \pm 5.39$ & $2.82(1.22,6.50)$ \\
\hline & $p$-value & $<0.001$ & $<0.001$ & \\
\hline \multirow[t]{4}{*}{ Emergency department visits } & Robust $(n=454)$ & $28(6.2)$ & $0.09 \pm 0.41$ & 1.00 \\
\hline & Pre-frail $(n=194)$ & $22(11.3)$ & $0.18 \pm 0.63$ & $1.10(0.55,2.21)$ \\
\hline & Frail $(n=53)$ & $16(30.2)$ & $0.57 \pm 1.01$ & $3.05(1.14,8.12)$ \\
\hline & $p$-value & $<0.001$ & $<0.001$ & \\
\hline \multirow[t]{4}{*}{ Day surgery attendances } & Robust $(n=454)$ & $18(4.0)$ & $0.06 \pm 0.37$ & 1.00 \\
\hline & Pre-frail $(n=194)$ & $10(5.2)$ & $0.09 \pm 0.61$ & $2.02(0.77,5.27)$ \\
\hline & Frail $(n=53)$ & $6(11.3)$ & $0.13 \pm 0.39$ & $6.41(1.33,30.92)$ \\
\hline & $p$-value & 0.060 & 0.062 & \\
\hline \multirow[t]{4}{*}{ Hospitalisations } & Robust $(n=454)$ & $13(2.9)$ & $0.04 \pm 0.27$ & 1.00 \\
\hline & Pre-frail $(n=194)$ & $20(10.3)$ & $0.14 \pm 0.47$ & $2.06(0.91,4.67)$ \\
\hline & Frail $(n=53)$ & $15(28.3)$ & $0.51 \pm 0.95$ & $6.72(2.14,21.11)$ \\
\hline & $p$-value & $<0.001$ & $<0.001$ & \\
\hline
\end{tabular}

${ }^{a} p$-values were obtained by chi-squared tests

${ }^{\mathrm{b}} \mathrm{p}$-values were obtained by Kruskal-Wallis $\mathrm{H}$ tests

IRR: Incidence rate ratio. Adjusted for age, female, Chinese, marital status, highest education level, living alone, self-reported money insufficiency, smoking status, multimorbidity, and any assistance required in ADLs

settings. While the frail elderly in the community had significantly higher proportion and number of SOC visits, ED visits, day surgery attendances and hospitalisations in the 6-month period prior to and after the baseline, their utilisation of public primary care services was lower relative to their pre-frail or robust peers.

Prior studies consistently reported that increasing frailty is associated with substantial increase in hospital admissions, measured either retrospectively or prospectively [8, $16,35]$. We also observed that the frail older adults in the study had more hospitalisations than their robust and prefrail peers, regardless whether the hospitalisations occurred prior to or after the baseline. Their association is persistent even after adjusting for the socio-demographics, multimorbidity and disability. Among the healthcare service utilisation in the five different care settings, our study found that frailty had the most significant impact on hospitalisations in both 6-month period prior to and after the baseline, which is consistent with findings reported in prior studies $[8,12,36]$. The association between frailty and hospitalisations indicates that frail elderly in Singapore tend to present to the healthcare system, especially tertiary care, when they are in a more severe stage of frailty [37].
Unlike prior studies which reported that frailty had a positive association with probability of use of primary care services in general practitioner clinics $[8,15,16$, 36], our study found frail individuals did not have higher risk of utilising more polyclinic services than their robust counterparts. Instead, the older adults who were in the pre-frail stage tended to use more polyclinic services. This suggests that when older adults deteriorate from robust health stage to pre-frail stage, their use of primary care services increase significantly; and when older adults are in a more severe stage of frailty, their needs may shift towards increased specialist care services. However, the results should be interpreted with caution as only about $20 \%$ of the total primary care services in Singapore are provided by polyclinics and the left $80 \%$ are provided by private general practitioners (GPs). Although polyclinics provide a relatively larger percentage of care for patients with chronic and more complex conditions, the omission of GP utilisation data in the RHS database makes it challenging to infer the association between frailty and the total primary care utilisation.

Although the association between frailty and healthcare utilisation of specialist outpatient care is less investigated compared with that of inpatient services, prior 
Table 4 Associations between frailty and healthcare utilisation in different settings during the 6-month period after the baseline

\begin{tabular}{|c|c|c|c|c|}
\hline Healthcare utilisation by setting & Frailty & Yes, n (\%) & Mean \pm SD & Adjusted IRR ${ }^{\mathrm{C}}(95 \% \mathrm{Cl})$ \\
\hline \multirow[t]{4}{*}{ Polyclinic visits } & Robust $(n=454)$ & $153(33.7)$ & $0.97 \pm 2.3$ & 1.00 \\
\hline & Pre-frail $(n=194)$ & $82(42.3)$ & $1.64 \pm 4.18$ & $1.54(1.08,2.19)$ \\
\hline & Frail $(n=53)$ & $20(37.7)$ & $1.11 \pm 1.82$ & $1.17(0.60,2.29)$ \\
\hline & $p$-value & $0.113^{a}$ & $0.080^{b}$ & \\
\hline \multirow[t]{4}{*}{ Specialist outpatient clinic visits } & Robust $(n=454)$ & $139(30.6)$ & $1.21 \pm 2.61$ & 1.00 \\
\hline & Pre-frail $(n=194)$ & $70(36.1)$ & $2.03 \pm 4.13$ & $1.48(0.96,2.27)$ \\
\hline & Frail $(n=53)$ & $31(58.5)$ & $5.08 \pm 7.32$ & $3.31(1.56,7.06)$ \\
\hline & $p$-value & $<0.001$ & $<0.001$ & \\
\hline \multirow[t]{4}{*}{ Emergency department visits } & Robust $(n=454)$ & $20(4.4)$ & $0.05 \pm 0.25$ & 1.00 \\
\hline & Pre-frail $(n=194)$ & $20(10.3)$ & $0.19 \pm 0.73$ & $2.55(1.25,5.20)$ \\
\hline & Frail $(n=53)$ & $16(30.2)$ & $0.47 \pm 0.82$ & $6.40(2.38,17.24)$ \\
\hline & $p$-value & $<0.001$ & $<0.001$ & \\
\hline \multirow[t]{4}{*}{ Day surgery attendances } & Robust $(n=454)$ & $24(5.3)$ & $0.06 \pm 0.31$ & 1.00 \\
\hline & Pre-frail $(n=194)$ & $11(5.7)$ & $0.09 \pm 0.47$ & $1.77(0.77,4.06)$ \\
\hline & Frail $(n=53)$ & $5(9.4)$ & $0.13 \pm 0.44$ & $5.75(1.28,25.78)$ \\
\hline & $p$-value & 0.468 & 0.450 & \\
\hline \multirow[t]{4}{*}{ Hospitalisations } & Robust $(n=454)$ & $11(2.4)$ & $0.03 \pm 0.17$ & 1.00 \\
\hline & Pre-frail $(n=194)$ & $19(9.8)$ & $0.12 \pm 0.41$ & $3.76(1.66,8.53)$ \\
\hline & Frail $(n=53)$ & $16(30.2)$ & $0.53 \pm 0.97$ & $13.11(4.90,35.04)$ \\
\hline & $p$-value & $<0.001$ & $<0.001$ & \\
\hline
\end{tabular}

${ }^{a} p$-values were obtained by chi-squared tests

b $p$-values were obtained by Kruskal-Wallis $\mathrm{H}$ tests

IRR: Incidence rate ratio. Adjusted for age, female, Chinese, marital status, highest education level, living alone, self-reported money insufficiency, smoking status, multimorbidity, and any assistance required in ADLs

studies do suggest that frailty has positive association with the use of specialist outpatient services [8, 38]. Our study provides additional support for their association, regardless whether the SOC visits occurred in 6 months prior to or after the baseline. This reflects that an increase in the degree of frailty among older adults corresponds with a greater need for comprehensive and specialised health care services [38, 39].

The association between frailty and number of surgical procedures performed in DS rooms were scarcely examined separately in prior studies. A relatively higher proportion and rate of DS attendances among frail individuals was observed in the study, with no significant difference in associations between 6 months prior to and after the baseline. However, we are unable to draw any causal conclusion between frailty and DS attendances. Although the degree of frailty prior to general surgery is increasingly used for risk-stratification, as most of the DS procedures are considered low-stress procedures for older adults, the findings in the study suggests that frail individuals have a greater need for day surgeries. As preoperative frailty is associated with adverse clinical outcomes even for low-stress procedures [40], comprehensive frailty screening should be conducted prior to surgical procedures not only for optimization before surgery but also for targeted interventions before and after surgery.

It has been well established that multimorbidity is bidirectionally associated with frailty [41] and greater healthcare utilisation [42]. A recent study suggests that frailty may be directly related to kidney function [43]. We also observed that CKD, which accounted for $20 \%$ of the multimorbidity, was also highly prevalent among frail groups (49\%). Our result showed consistent associations between multimorbidity and number of polyclinics and SOC visits in both 6-month period prior to and after the baseline. However, the associations between multimorbidity and number of ED and DS attendances observed in these two periods were controversial, and no association was observed between multimorbidity and hospitalisation. This could probably be explained by the prevalence of individual chronic conditions that were used to define multimorbidity. As dyslipidaemia and high blood pressure were the most prevalent chronic conditions among the study population with prevalence of 66 and $64 \%$ respectively, the multimorbidity was highly attributed to either of the two conditions (high blood pressure $84 \%$ and dyslipidaemia $88 \%$ ), which are 
usually monitored in primary care settings or SOCs. While polytherapy is often mandatory in the management of multimorbidity [44], therapeutic decisions for elderly people should be approached cautiously as a more elevated polytherapy index is associated with the development of frailty [45] and increased healthcare utilisation.

\section{Strengths and limitations}

To the best of our knowledge, this is the first study investigating the association between frailty and patterns of healthcare utilisation in different care settings in Singapore. We examined the association using both retrospective and prospective utilisation data and found consistent relationship between frailty and healthcare utilisation in respective settings. However, as there is still some uncertainty whether frailty occurred before or after healthcare utilisation, the claim of causal inferences is limited.

The analyses presented in the study used number of hospitalisations to capture the inpatient utilisation. It is acknowledged that number of hospitalisations is a partial indicator of inpatient utilisation, length of stay, which also reflects another important aspect of inpatient utilisation [46], was not measured.

The healthcare utilisation data were derived from one RHS database, as such, healthcare utilisation in other RHS, GPs, private SOCs and hospitals, as well as home care services provided by Voluntary Welfare Organisations were not included. This may cause underestimation of the association between frailty and healthcare utilisation. However, as the participants were the residents in the region with their health entrusted to the respective RHS, the majority of their utilisation in public healthcare services should have been captured and serve the purpose of understanding the patterns of service delivery in these five care settings for older people with different frailty status within the defined geographical region served by the RHS.

\section{Conclusions}

Frailty was positively associated with SOC visits, ED visits, DS attendances and hospitalisations during 6month period prior to or after the baseline among community-dwelling older adults. Frail individuals tended to have higher rate of SOC and ED visits, DS attendances and hospitalisations compared to their robust counterpart. As frailty is a potentially reversible health state with early screening and intervention, identifying the pre-frail and frail elderly in the community, and providing effective interventions at early stage could be an effective strategy of reducing or delaying utilisation of secondary and tertiary care services.

\section{Supplementary information}

Supplementary information accompanies this paper at https://doi.org/10. 1186/s12877-020-01800-8.

\section{Additional file 1.}

\section{Abbreviations}

ADLs: Activities of daily living; ANOVA: Analysis of covariance; BMl: Body mass index; Cl: Confidence interval; CKD: Chronic kidney disease; COPD: Chronic obstructive pulmonary disease; DS: Day surgery; ED: Emergency departments; GPs: General practitioners; IRR: Incidence rate ratio; MNA: Mini Nutritional Assessment; NHG: National Healthcare Group; NRIC: National Registration Identity Card; OR: Odds ratio; PHI: Population Health Index; RHS: Regional healthcare system; SD: Standard deviation; SOC: Specialist outpatient clinic

\section{Acknowledgements}

The authors are thankful to Mr. Teow Kiok Liang for granting permission to link PHI survey data with RHS utilisation data.

\section{Authors' contributions}

LG conceived and designed the study, analysed and interpreted the data, drafted the first version of the article and revised it. CWY conceptualised the revised FRAIL scale, interpreted the data, and revised the article. $\mathrm{BHH}$ obtained funding, interpreted the data and revised the article. WST conceived the study, interpreted the data, reviewed and substantively revised the article. All authors read and have approved the submitted version.

\section{Funding}

This work was supported by National Healthcare Group Pte Ltd. in the form of salaries for all authors. The funder had no role in / influence on study design, data collection and analysis, decision to publish, or preparation of the manuscript.

\section{Availability of data and materials}

The datasets used and/or analysed during the current study are available from the corresponding author on reasonable request.

\section{Ethics approval and consent to participate}

The PHI study was approved by the ethics review committee of the National Healthcare Group Domain Specific Review Board (Reference Number: 2015/ 00269). This ethic approval includes linking PHI survey data with utilisation data. The permission to link PHI survey data with utilisation data in RHS database has been obtained from Principle Investigators of the two studies. Written informed consent was obtained from all individual participants after they were being informed about the study objectives and the safeguards put in place so that confidentiality of the collected data is maintained.

\section{Consent for publication}

Not applicable.

Competing interests

The authors declare that they have no competing interests.

\section{Author details}

${ }^{1}$ Health Services \& Outcomes Research, National Healthcare Group Pte Ltd, 3 Fusionopolis Link, \#03-08 Nexus@one-north (South Lobby), Singapore 13854, Singapore. ${ }^{2}$ Geriatric Education and Research Institute, Singapore, Singapore.

Received: 6 May 2020 Accepted: 28 September 2020

Published online: 06 October 2020

References

1. Howlett SE, Rockwood K. New horizons in frailty: ageing and the deficitscaling problem. Age Ageing. 2013;42:416-23.

2. Collard RM, Boter H, Schoevers RA, Oude Voshaar RC. Prevalence of frailty in community-dwelling older persons: a systematic review. J Am Geriatr Soc. 2012;60:1487-92.

3. WHO WHO. Facts about ageing. Geneva: WHO; 2014. http://www.who.int/ ageing/about/facts/en/. Accessed 18 Feb 2019. 
4. Buckinx F, Rolland Y, Reginster J-Y, Ricour C, Petermans J, Bruyère O. Burden of frailty in the elderly population: perspectives for a public health challenge. Arch Public Health. 2015;73:19.

5. Department of Statistics. Singapore residents by age group, ethnic group and sex. Population and population structure. 2020. http://www. tablebuilder.singstat.gov.sg/publicfacing/createDataTable.action?refld=14911.

6. United Nations, Department of Economic and Social Affairs, Population Division. World population ageing 2017 - highlights (ST/ESA/SER.A/397). New York: the United Nations; 2017.

7. Hajek A, Bock J-O, Saum K-U, Matschinger H, Brenner H, Holleczek B, et al. Frailty and healthcare costs-longitudinal results of a prospective cohort study. Age Ageing. 2018;47:233-41.

8. Roe L, Normand C, Wren M-A, Browne J, O'Halloran AM. The impact of frailty on healthcare utilisation in Ireland: evidence from the Irish longitudinal study on ageing. BMC Geriatr. 2017;17:203. https://doi.org/10. 1186/s12877-017-0579-0.

9. Ministry of Health. Reorganisation of healthcare system into three integrated clusters to better meet future healthcare needs. Singapore: Ministry of Health Singapore; 2017. News Highlights. https:/www.moh.gov.sg/news-highlights/ details/reorganisation-of-healthcare-system-into-three-integrated-clusters-tobetter-meet-future-healthcare-needs. Accessed 25 Mar 2020.

10. Lim WS, Wong SF, Leong I, Choo P, Pang WS. Forging a frailty-ready healthcare system to meet population ageing. Int J Environ Res Public Health. 2017;14:e1448.

11. Dent E, Hoon E, Karnon J, Newbury J, Kitson A, Beilby J. Frailty and health service use in rural South Australia. Arch Gerontol Geriatr. 2016;62:53-8.

12. Hoeck S, François G, Geerts J, Van der Heyden J, Vandewoude M, Van Hal G. Health-care and home-care utilization among frail elderly persons in Belgium. Eur J Pub Health. 2012;22:671-7.

13. Rochat S, Cumming RG, Blyth F, Creasey H, Handelsman D, Le Couteur DG, et al. Frailty and use of health and community services by communitydwelling older men: the Concord Health and Ageing in Men Project. Age Ageing. 2010;39:228-33.

14. Ensrud KE, Kats AM, Schousboe JT, Taylor BC, Cawthon PM, Hillier TA, et al. Frailty phenotype and healthcare costs and utilization in older women. J Am Geriatr Soc. 2018;66:1276-83.

15. Simpson KN, Seamon BA, Hand BN, Roldan CO, Taber DJ, Moran WP, et al. Effect of frailty on resource use and cost for Medicare patients. J Comp Eff Res. 2018;7:817-25.

16. Gobbens RJJ, van Assen MALM, Luijkx KG, Schols JMGA. Predictive validity of the Tilburg frailty indicator: disability, health care utilization, and quality of life in a population at risk. The Gerontologist. 2012;52:619-31.

17. Chan A, Raman P, Ma S, Malhotra R. Loneliness and all-cause mortality in community-dwelling elderly Singaporeans. Demogr Res. 2015;S15:1361-82.

18. Hogan DB, Maxwell CJ, Afilalo J, Arora RC, Bagshaw SM, Basran J, et al. A scoping review of frailty and acute care in middle-aged and older individuals with recommendations for future research. Can Geriatr J. 2017; 20:22-37.

19. Ge L, Ong R, Yap CW, Heng BH. Effects of chronic diseases on health-related quality of life and self-rated health among three adult age groups. Nurs Health Sci. 2018;21(2):214-22

20. Ge L, Yap CW, Heng BH. Sex differences in associations between multimorbidity and physical function domains among community-dwelling adults in Singapore. PLoS One. 2018;13:e0197443.

21. Ge L, Yap CW, Ong R, Heng BH. Social isolation, loneliness and their relationships with depressive symptoms: a population-based study. PLoS One. 2017;12:e0182145.

22. Kish L. A procedure for objective respondent selection within the household. J Am Stat Assoc. 1949:44:380-7.

23. Abellan van Kan G, Rolland YM, Morley JE, Vellas B. Frailty: toward a clinical definition. J Am Med Dir Assoc. 2008;9:71-2.

24. Morley JE, Malmstrom TK, Miller DK. A simple frailty questionnaire (FRAIL) predicts outcomes in middle aged African Americans. J Nutr Health Aging. 2012;16:601.

25. Gunapal PPG, Kannapiran P, Teow KL, Zhu Z, Xiaobin You A, Saxena N, et al. Setting up a regional health system database for seamless population health management in Singapore. Proc Singapore Healthc. 2016;25:27-34.

26. Peters LL, Burgerhof JGM, Boter H, Wild B, Buskens E, Slaets JPJ. Predictive validity of a frailty measure (GFI) and a case complexity measure (IM-E-SA) on healthcare costs in an elderly population. J Psychosom Res. 2015;79:404-11.
27. Kuuire VZ, Bisung E, Rishworth A, Dixon J, Luginaah I. Health-seeking behaviour during times of illness: a study among adults in a resource poor setting in Ghana. J Public Health. 2015;38:fdv176.

28. Low L, Tay W, Ng M, Tan S, Liu N, Lee K. Frequent hospital admissions in Singapore: clinical risk factors and impact of socioeconomic status. Singap Med J. 2018:59:39-43.

29. Fried LP, Tangen CM, Walston J, Newman AB, Hirsch C, Gottdiener J, et al. Frailty in older adults: evidence for a phenotype. J Gerontol Med Sci. 2001 56A:M146-56. https://doi.org/10.1093/gerona/56.3.M146.

30. Fried LP, Ferrucci L, Darer J, Williamson JD, Anderson G. Untangling the concepts of disability, frailty, and comorbidity: implications for improved targeting and care. J Gerontol A Biol Sci Med Sci. 2004;59:255-63.

31. Shah S, Vanclay F, Cooper B. Improving the sensitivity of the Barthel index for stroke rehabilitation. J Clin Epidemiol. 1989;42:703-9.

32. Diehr $P$, Yanez D, Ash A, Hornbrook M, Lin DY. Methods for analyzing health care utilization and costs. Annu Rev Public Health. 1999;20:125-44.

33. Sarma S, Simpson W. A microeconometric analysis of Canadian health care utilization. Health Econ. 2006;15:219-39.

34. Payne EH, Gebregziabher M, Hardin JW, Ramakrishnan V, Egede LE. An empirical approach to determine a threshold for assessing overdispersion in Poisson and negative binomial models for count data. Commun Stat Simul Comput. 2018:47:1722-38

35. Ng TP, Feng L, Nyunt MSZ, Larbi A, Yap KB. Frailty in older persons: multisystem risk factors and the Frailty Risk Index (FRI). J Am Med Dir Assoc. 2014;15:635-42.

36. Han L, Clegg A, Doran T, Fraser L. The impact of frailty on healthcare resource use: a longitudinal analysis using the clinical practice research Datalink in England. Age Ageing. 2019;48:665-71.

37. Chen CY, Gan P, How CH. Approach to frailty in the elderly in primary care and the community. Singap Med J. 2018;59:240-5.

38. Tan LF, Lim ZY, Choe R, Seetharaman S, Merchant R. Screening for frailty and sarcopenia among older persons in medical outpatient clinics and its associations with healthcare burden. J Am Med Dir Assoc. 2017;18:583-7.

39. Clegg A, Young J, lliffe S, Rikkert MO, Rockwood K. Frailty in elderly people. Lancet. 2013;381:752-62.

40. Shinall MC, Arya S, Youk A, Varley P, Shah R, Massarweh NN, et al. Association of preoperative patient frailty and operative stress with postoperative mortality. JAMA Surg. 2020;155:e194620.

41. Vetrano DL, Palmer K, Marengoni A, Marzetti E, Lattanzio F, RollerWirnsberger $\mathrm{R}$, et al. Frailty and multimorbidity: a systematic review and meta-analysis. J Gerontol A Biol Sci Med Sci. 2019;74:659-66.

42. Palladino R, Tayu Lee J, Ashworth M, Triassi M, Millett C. Associations between multimorbidity, healthcare utilisation and health status: evidence from 16 European countries. Age Ageing. 2016;45:431-5.

43. Coppolino G, Bolignano D, Gareri P, Ruberto C, Andreucci M, Ruotolo G, et al. Kidney function and cognitive decline in frail elderly: two faces of the same coin? Int Urol Nephrol. 2018;50:1505-10.

44. Gujjarlamudi HB. Polytherapy and drug interactions in elderly. J Midlife Health. 2016;7:105-7.

45. Gutiérrez-Valencia M, Izquierdo M, Cesari M, Casas-Herrero Á, Inzitari M, Martínez-Velilla N. The relationship between frailty and polypharmacy in older people: a systematic review. Br J Clin Pharmacol. 2018;84:1432-44.

46. Hubbard RE, Peel NM, Samanta M, Gray LC, Mitnitski A, Rockwood K. Frailty status at admission to hospital predicts multiple adverse outcomes. Age Ageing. 2017;46:801-6.

\section{Publisher's Note}

Springer Nature remains neutral with regard to jurisdictional claims in published maps and institutional affiliations. 